\title{
Practical Design Approaches for Assessing Parallelism in Dose Response Modelling
}

Timothy O'Brien

Loyola University Chicago, tobrie1@luc.edu

Jack Silcox

Loyola University Chicago

Follow this and additional works at: https://ecommons.luc.edu/math_facpubs

Part of the Mathematics Commons

\section{Author Manuscript}

This is a pre-publication author manuscript of the final, published article.

\section{Recommended Citation}

O'Brien, Timothy and Jack Silcox, "Practical Design Approaches for Assessing Parallelism in Dose Response Modelling" in Contemporary Biostatistics with Biopharmaceutical Applications, Zhang, L., Chen, D.-G., Jiang, H., Li, G., Quan, H. (Eds.), Springer, 2019.

This Book Chapter is brought to you for free and open access by the Faculty Publications and Other Works by Department at Loyola eCommons. It has been accepted for inclusion in Mathematics and Statistics: Faculty Publications and Other Works by an authorized administrator of Loyola eCommons. For more information, please contactecommons@luc.edu. cc) (i) $\Theta$

This work is licensed under a Creative Commons Attribution-Noncommercial-No Derivative Works 3.0 License. (c) Springer Nature Switzerland AG, 2019. 


\section{Practical Design Approaches for Assessing Parallelism in Dose Response Modelling}

Timothy E. O'Brien ${ }^{1,2}$ and Jack Silcox ${ }^{1,3}$

${ }^{1}$ Department of Mathematics and Statistics, Loyola University Chicago, Chicago, IL 60660 USA

${ }^{2}$ Institute of Environmental Sustainability, Loyola University Chicago, Chicago, IL 60660 USA

${ }^{3}$ University of Utah, Cognitive and Neural Science PhD Program, Salt Lake City, UT 84132 USA

Corresponding e-mail: tobrie1@luc.edu

Keywords: dose response, geometric design, lack of fit, logistic regression, mechanism of action, median lethal concentration, model misspecification, parallelism, robust optimal design, uniform design.

Index terms: bioassay, compound design criteria, D-efficiency, dose response, EC50, geometric design, goodness of fit, immunology, lack of fit, LD50, logistic regression, mechanism of action, median lethal concentration, microbiology, model misspecification, nonlinear regression, optimal design, parallelism, pharmacology, profile likelihood confidence interval, quantile response, simulation, relative potency, robustness, similar compounds, subset design, toxicology, uniform design, virology. 


\section{Practical Design Approaches for Assessing Parallelism in Dose Response Modelling}

Timothy E. O'Brien ${ }^{1,2}$ and Jack Silcox ${ }^{1,3}$

${ }^{1}$ Department of Mathematics and Statistics, Loyola University Chicago, Chicago, IL 60660 USA

${ }^{2}$ Institute of Environmental Sustainability, Loyola University Chicago, Chicago, IL 60660 USA

${ }^{3}$ University of Utah, Cognitive and Neural Science PhD Program, Salt Lake City, UT 84132 USA

\section{Introduction}

Scientific researchers in biomedicine, pharmaceutical science and toxicology often face situations in which binary logistic regression model fits are used to compare two drugs or substances, often by means of comparisons of median doses or concentrations (EC50 or LD50). Applications are given in works spanning early bioassay findings of Finney $(1971,1978)$ to more recent results in Rich $(2013)$ and Gupta and Vale (2017). Furthermore, Wheeler et al. (2006) underscores the caution that instead of examining for overlap in separate EC50 confidence intervals, testing is best based on estimation and confidence intervals associated with the relative potency parameter. Notably, before fitting such curves and testing for differing potencies, an important requirement is that these dose response curves be parallel. As such, various works have introduced meaningful means to assess parallelism in logistic regression settings, including Gottschalk and Dunn (2005), Jonkman and Sidak (2009), Novick et al. (2012), Yang and Zhang (2012), Yang et al. (2012), Fleetwood et al. (2015) and Sidak and Jonkman (2016).

So as to efficiently test for common slopes of drug curves, our focus here is on developing robust, efficient and practical design strategies in the assessment of dose response curve parallelism.

Overviews of optimal design theory and applications are given in O'Brien and Funk (2003) and Atkinson et al. (2007). Additionally, classical experimental design strategies for binary logistic regression models are given in Abdelbasit and Plackett (1983), Minkin (1987) and Kalish (1990), and model-robust design approaches are given and explored in Atkinson (1972), O'Brien (2005), O'Brien (2016), O'Brien (2018), O'Brien and Rawlings (1996), O'Brien and Lim (2018), O'Brien et al. (2009) and O'Brien et al. (2010).

\section{Assessing Parallelism in Dose Response}

In situations where the outcome variable is a percentage derived from binary outcomes - such as percentage mortality in a microbiology or toxicology experiment - the two-parameter (binomial, logitlink) logistic model is often used to model the dose-response data. This generalized nonlinear model is written

$$
\log \left(\frac{\pi}{1-\pi}\right)=\eta=\beta(x-\gamma)
$$

Here, $\pi$ is the probability of outcome (e.g., mortality), $\beta$ is the slope, $x$ is the concentration or dose of the drug or compound, and $\gamma$ is the EC50/LD50 parameter so that $x=\gamma$ coincides with $\pi=\frac{1}{2}$ (or $50 \%$ chance of death). Equivalent to (1) is the expression $\pi=\frac{e^{\beta(x-\gamma)}}{1+e^{\beta(x-\gamma)}}$. This model can be extended to simultaneously model two curves (such as corresponding to two viruses or drugs, labelled " $A$ " and " $B$ "), as graphed in Figure 1, by modifying the right-hand side in (1) to be 


$$
\eta= \begin{cases}\beta\left(x-\gamma_{A}\right), & \operatorname{drug} A \\ (\beta+\delta)\left(x-\gamma_{B}\right), & \text { drug } B\end{cases}
$$

$\ln (2), \beta$ is the slope of the drug A curve, $(\beta+\delta)$ is the slope of the drug $\mathrm{B}$ curve, and the respective EC50's are $\gamma_{A}$ and $\gamma_{B}$ for drugs A and B. Our goal in fitting this model is to fit the respective curves with particular focus on the difference-of-slopes parameter $\delta$. Both curves can be written in a single model as $\pi=\frac{e^{\eta}}{1+e^{\eta}}$ with $\eta$ here given in (2). It is important to point out that both curves are fit - and indeed designed - simultaneously since they share the joint parameter $\beta$. These curves are plotted in Figure $1-$ where $\pi$ is the percent response - using parameter values $\beta=0.30, \delta=0, \gamma_{A}=11.0, \gamma_{B}=14.5$.

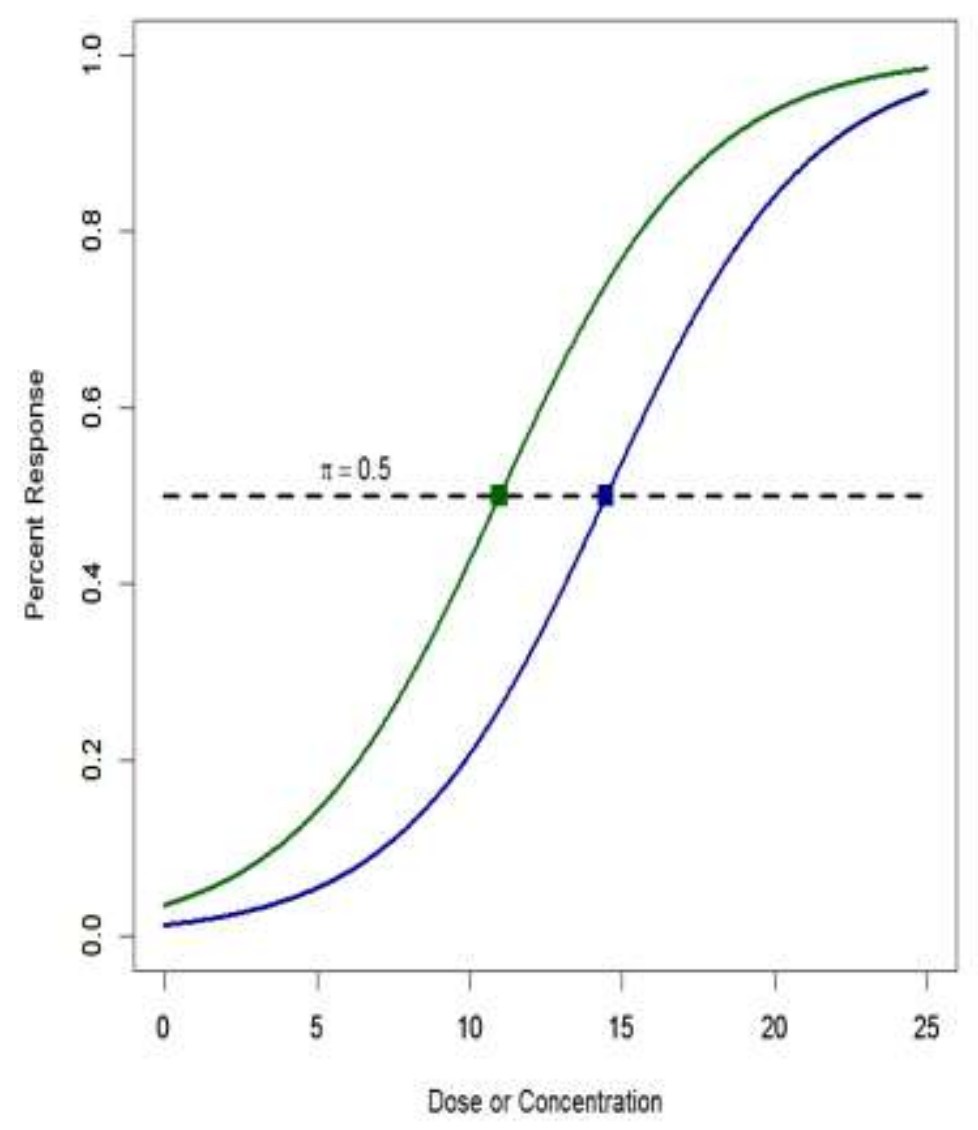

Figure 1. Plot of parallel ( $\delta=0$ ) binary logistic curves with vertical axis corresponding to probability of outcome or mortality, slope $\beta=0.30$ and EC50's $\gamma_{A}=11.0$ (left curve) and $\gamma_{B}=14.5$ (right curve); here, relative potency is then estimated to be $11.0 / 14.5=1.32$. Solid squares indicate EC50's as points where the respective curves cross cut-line, $\pi=1 / 2$.

\section{Optimal Design Background}


Approximate designs, denoted $\xi$, are written

$$
\xi=\left\{\begin{array}{llll}
x_{1} & x_{2} & \ldots & x_{n} \\
\omega_{1} & \omega_{2} & \ldots & \omega_{n}
\end{array}\right\}
$$

The $\omega_{i}$ are non-negative design weights which sum to one, and the $x_{i}$ are design points (i.e., concentrations) that belong to the design space and are not necessarily distinct. Further, the $p$ model parameters are stacked into the p-vector $\boldsymbol{\theta}^{\boldsymbol{T}}=\left(\beta, \gamma_{A}, \gamma_{B}, \delta\right)$. In the constant-variance Normal/Gaussian setting with linear or nonlinear normal model function $\eta(x, \boldsymbol{\theta})$, the $n \times p$ Jacobian matrix is $\boldsymbol{V}=\frac{\partial \eta}{\partial \theta}$ and with $\boldsymbol{\Omega}=\operatorname{diag}\left\{\omega_{1}, \omega_{2}, \ldots, \omega_{n}\right\}$, the $p \times p$ (Fisher) information matrix is written

$$
M(\xi, \theta)=V^{T} \Omega V
$$

Atkinson et al (2007) demonstrates that for binomial logistic models in general, the information matrix for the relative-potency logistic model considered here has the same form as in (4) with a modification of the weight matrix $\boldsymbol{\Omega}$. Specifically, $\boldsymbol{\Omega}$ in this case is a diagonal matrix with $i^{\text {th }}$ diagonal element $\omega_{i} \pi_{i}\left(1-\pi_{i}\right)$, where $\pi_{i}$ is the success probability. In regression settings, since the (asymptotic) variance of the maximum-likelihood estimator $\widehat{\boldsymbol{\theta}}_{M L E}$ is proportional to $\boldsymbol{M}^{-1}(\xi, \boldsymbol{\theta})$, designs are often chosen to minimize some (convex) function of $\boldsymbol{M}^{-1}(\xi, \boldsymbol{\theta})$. For example, designs which minimize the determinant of $\boldsymbol{M}^{-1}$ - and equivalently which maximize the determinant of $\boldsymbol{M}$ - are called D-optimal.

Since our focus is on the difference-of-slopes parameter $(\delta)$ more so than the other parameters, we partition the Fisher information matrix as

$$
M=\left[\begin{array}{ll}
M_{11} & M_{12} \\
M_{21} & M_{22}
\end{array}\right]
$$

Each sub-matrix $\boldsymbol{M}_{\boldsymbol{i} \boldsymbol{j}}$ is of dimension $p_{i} \times p_{j}$ for $i, j=1,2$, and $p_{1}+p_{2}=p$. The parameter vector is also partitioned, $\boldsymbol{\theta}=\left(\begin{array}{l}\boldsymbol{\theta}_{1} \\ \boldsymbol{\theta}_{2}\end{array}\right)$ with $\boldsymbol{\theta}_{1}$ (the so-called nuisance parameters) of dimension $p_{1} \times 1$ and $\boldsymbol{\theta}_{2}$ (the parameter of interest) of dimension $p_{2} \times 1$. In the current situation, $\boldsymbol{\theta}_{1}^{T}=\left(\beta, \gamma_{A}, \gamma_{B}\right)$ so $p_{1}=3$ and $\theta_{2}=\delta$ so $p_{2}=1$. As outlined in Atkinson et al. (2007), $D_{s}\left(\boldsymbol{\theta}_{2}\right)$ subset designs maximize

$$
\left|M_{22}-M_{21} M_{11}^{-1} M_{12}\right|=\frac{|M|}{\left|M_{11}\right|}
$$

Because of problems associated with subset designs, some authors suggest combining the subset and full parameter criteria so that for a given $\alpha \in\left[\frac{p_{2}}{p}, 1\right]$, designs be chosen to maximize the compound objective function (see O'Brien (2005) and Atkinson et al. (2007)),

$$
\Phi_{\alpha}(\xi, \boldsymbol{\theta})=\frac{1-\alpha}{p_{1}} \log \left|\boldsymbol{M}_{\mathbf{1 1}}\right|+\frac{\alpha}{p_{2}} \log \left|\boldsymbol{M}_{\mathbf{2 2}}-\boldsymbol{M}_{\mathbf{2 1}} \boldsymbol{M}_{\mathbf{1 1}}^{-1} \boldsymbol{M}_{\mathbf{1 2}}\right|
$$


A generalized inverse is used in (7) when $\boldsymbol{M}_{\mathbf{1 1}}$ is not invertible. This objective function ranges from the $\mathrm{D}$-optimal criterion for $\alpha=\frac{p_{2}}{p}$ to the subset design criterion (for $\delta$ ) in (6) for $\alpha=1$. For a given choice of $\alpha \in\left[\frac{p_{2}}{p}, 1\right]$, we call designs that maximize (7) $D_{\alpha}$-optimal designs.

Extending the results given in O'Brien (2016), our results here for the two-logistic situation in (2) and Figure 1 validate that the optimal values of $s=e^{\eta}$ for $D_{\alpha}$-optimal designs satisfy the expression,

$$
(1+s)+A(1-s) \log (s)=0
$$

In (8), $A=\frac{\alpha p_{1}+3(1-\alpha) p_{2}}{2\left(\alpha p_{1}+(1-\alpha) p_{2}\right)}$. The $s$-values which solve this expression are observed to be reciprocals, and this has been demonstrated to be the case in other situations when working with logistic regression (see O'Brien (2016), O'Brien and $\operatorname{Lim}$ (2018), and O'Brien et al. (2009)). As in these other situations, Equation (8) has exactly two roots for $\alpha \in\left[\frac{p_{2}}{p}, 1\right]$. For example, for $\alpha=\frac{p_{2}}{p}=0.25$ (i.e., the D-optimality criterion), $A=1$ and solution of (8) gives $s_{1}=0.2137$ (and $\pi_{1}=\frac{s_{1}}{1+s_{1}}=0.1760$ ) and $s_{2}=4.6805$ (and $\pi_{2}=0.8240$ ). As $\alpha$ approaches unity (i.e., the $D_{s}(\delta)$ subset design), we obtain $A=\frac{1}{2}, s_{1}=0.0908$ (and $\pi_{1}=0.0832$ ) and $s_{2}=11.0161$ (and $\pi_{2}=0.9168$ ). Since the $s$ values here are reciprocals, the corresponding values of $\pi$ necessarily sum to one.

Figure 2 shows the $D$-optimal design points along with the corresponding cut-lines at $\pi_{1}=0.1760$ and $\pi_{2}=0.8240$. The values of the design support points are obtained using the relations $x=\gamma_{A} \pm$ $\frac{1}{\beta} \log \left(s_{1}\right)$ for drug A (left curve in Figure 2) and $x=\gamma_{B} \pm \frac{1}{(\beta+\delta)} \log \left(s_{1}\right)$ for drug B (right curve in Figure 2). Thus, for the specific parameter values used here, the support points are $x=5.8554,16.1447$ for drug A (left curve) and $x=9.3554,19.6447$ for drug B (right curve). 


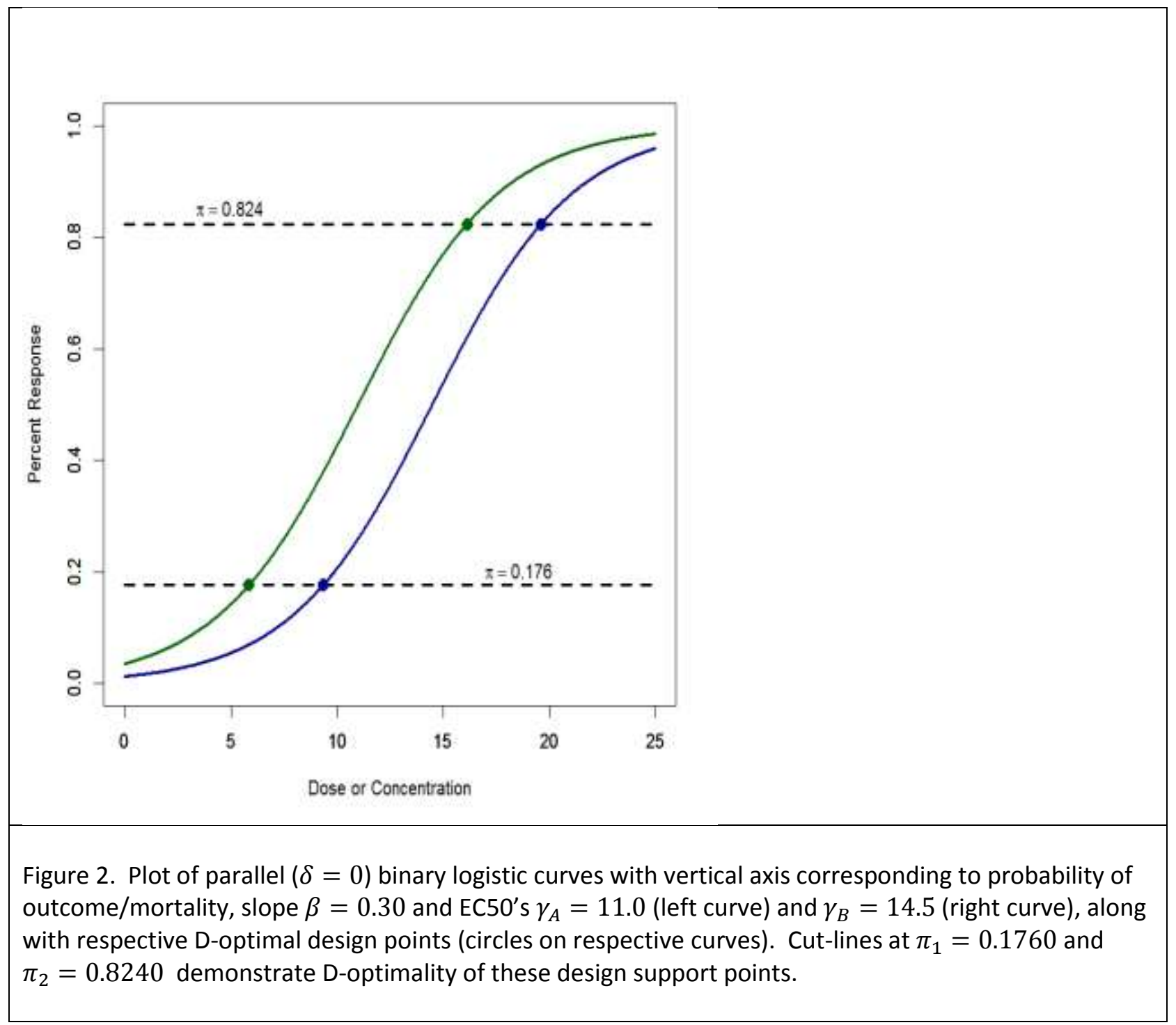

A measure of the distance between an arbitrary design $\xi$ and a D-optimal design $\xi_{D}^{*}$ is the D-efficiency $(D E F F)$ given by the expression $\left(\frac{|\boldsymbol{M}(\xi)|}{\left|\boldsymbol{M}\left(\xi_{D}^{*}\right)\right|}\right)^{1 / p}$ (see O'Brien and Funk (2003) and Atkinson et al. (2007)). A similar D-efficiency expression $\left(D E F F_{S}\right)$ can be given for subset efficiency in (6) but using $\mid \boldsymbol{M}_{\mathbf{2 2}}$ $\boldsymbol{M}_{\mathbf{2 1}} \boldsymbol{M}_{\mathbf{1 1}}^{-1} \boldsymbol{M}_{12} \mid$ in place of $|\boldsymbol{M}|$ in both numerator and denominator and raised to the power $\left(1 / p_{2}\right)$ instead of $(1 / p)$. For the current situation, full and subset efficiencies of the D-optimal and the $D_{S}(\delta)$ subset design are given in Table 1 . Note that as one shifts from the D-optimal to the $D_{S}(\delta)$ subset design (i.e., as $\alpha$ increases from 0.25 to 1.0), the design support points spread out away from the EC50s since the proportion cut lines (see Figure 2) drop from $\pi=0.1760$ to $\pi=0.0832$ and increase from $\pi=0.8240$ to $\pi=0.9168$. Also, as we shift from the D-optimal to the subset design, as expected we note the decrease in the variance term (diagonal term in $\boldsymbol{M}^{-1}$ ) associated with $\delta$ of over $20 \%$ (from 1.0419 to 0.8196 ) but also the approximately $90 \%$ increase in the variance terms associated with the $\gamma$ (EC50) terms (from 153.2 to 291.3). Finally, in noting the efficiency values of these two designs, one readily sees the trade-off nature in that as one efficiency increases, the other decreases, and vice versa. 
Table 1. $D$ - and $D_{S}(\delta)$-optimal designs in the parallel logistic setting: design support points and corresponding proportions, associated variance estimates for parameter values, and $D$ - and subset-efficiencies.

\begin{tabular}{|c|c|c|c|c|c|c|c|}
\hline$\alpha$ & Design & $\begin{array}{c}\text { Drug A } \\
\text { support } \\
\text { points }\end{array}$ & $\begin{array}{c}\text { Drug B } \\
\text { support } \\
\text { points }\end{array}$ & $\begin{array}{c}\text { Proportion } \\
\text { values }\end{array}$ & $\begin{array}{c}\text { Diagonal elements of } \\
\boldsymbol{M}^{-1} \text { corresponding to } \\
\boldsymbol{\theta}^{\boldsymbol{T}}=\left(\beta, \gamma_{A}, \gamma_{B}, \delta\right)\end{array}$ & $D E F F$ & $D E F F_{S}$ \\
\hline 0.25 & $D$-optimal & $\begin{array}{c}x_{1}=5.8554 \\
x_{2}=16.1447\end{array}$ & $\begin{array}{c}x_{1}=9.3554 \\
x_{2}=19.6447\end{array}$ & $\begin{array}{c}\pi_{1}=0.1760 \\
\pi_{2}=0.8240\end{array}$ & $\begin{array}{c}0.5210,153.2, \\
153.2,1.0419\end{array}$ & 1.00 & 0.787 \\
\hline \multirow{2}{*}{1.0} & $\begin{array}{c}D_{S}(\delta) \\
\text { subset- } \\
\text { optimal }\end{array}$ & $\begin{array}{c}x_{1}=3.0024 \\
x_{2}=18.9977\end{array}$ & $\begin{array}{c}x_{1}=6.5019 \\
x_{2}=22.4977\end{array}$ & $\begin{array}{c}\pi_{1}=0.0832 \\
\pi_{2}=0.9168\end{array}$ & $\begin{array}{c}0.4098,291.3, \\
291.3,0.8196\end{array}$ & 0.818 & 1.00 \\
\hline
\end{tabular}

Translating efficiencies into sample size requirements, note that an efficiency of 0.80 of a given design relative to an optimal design translates to $1 / 0.80=1.25$. So, a sample size $25 \%$ higher for the lessefficient design is needed (compared with the optimal design) to yield equivalent information.

The above advantages in terms of efficiency notwithstanding, optimal designs are often only used as a starting point since they often have shortcomings. In most practical situations, optimal designs for $p$ parameter model functions comprise only $p$ support points; this is observed here since the D-optimal designs for the two-parameter logistic curves graphed in Figure 2 have only two support points for each curve/drug. As such, these designs provide little or no ability to test for lack of fit of the assumed model. As a result, researchers often desire near-optimal, so-called "robust", designs which have extra support points that can then be used to test for model adequacy. Another important disadvantage of the optimal designs plotted in Figure 2 is that practitioners typically wish to use the same concentration values for both drugs. In the next section, we introduce and explore very useful strategies to obtain near-optimal, robust designs which address these shortcomings.

\section{Robust Design Approaches}

In this section, we propose practical experimental design strategies addressing the lack-of-fit and sameconcentration considerations raised in the previous section. The requirement that the same concentrations be used for both dose-response curves leads us to consider finding the corresponding same-concentration-restricted $D$ - and $D_{S}(\delta)$-optimal designs, and this is done in the following section. In the subsequent sections, we introduce and examine so-called reflection designs based upon the original optimal designs, as well as geometric and uniform designs.

\subsection{Same-Concentration Designs}

In the current illustration - as well as in other situations with the two-drug logistic model in (2) - the same-concentration-restricted $D$-optimal design comprises two support points (for both drugs), and this is demonstrated in Figure 3 and Table 2. As demonstrated in Figure 3, the optimal proportions of $\pi_{1 A}=0.2583$ and $\pi_{2 A}=0.8914$ for drug $\mathrm{A}$ and $\pi_{1 B}=0.1086$ and $\pi_{2 B}=0.7417$ for drug $\mathrm{B}$ are such 
that (1) the lower proportions $\left(\pi_{1 A}\right.$ and $\pi_{1 B}$ ) straddle the cut-line of $\pi=0.176$ from Figure 2 and the upper proportions $\left(\pi_{2 A}\right.$ and $\left.\pi_{2 B}\right)$ straddle the cut-line of $\pi=0.824$ from Figure 2 , and (2) are reciprocally related via $\pi_{2 A}=1-\pi_{1 B}$ and $\pi_{2 B}=1-\pi_{1 A}$.

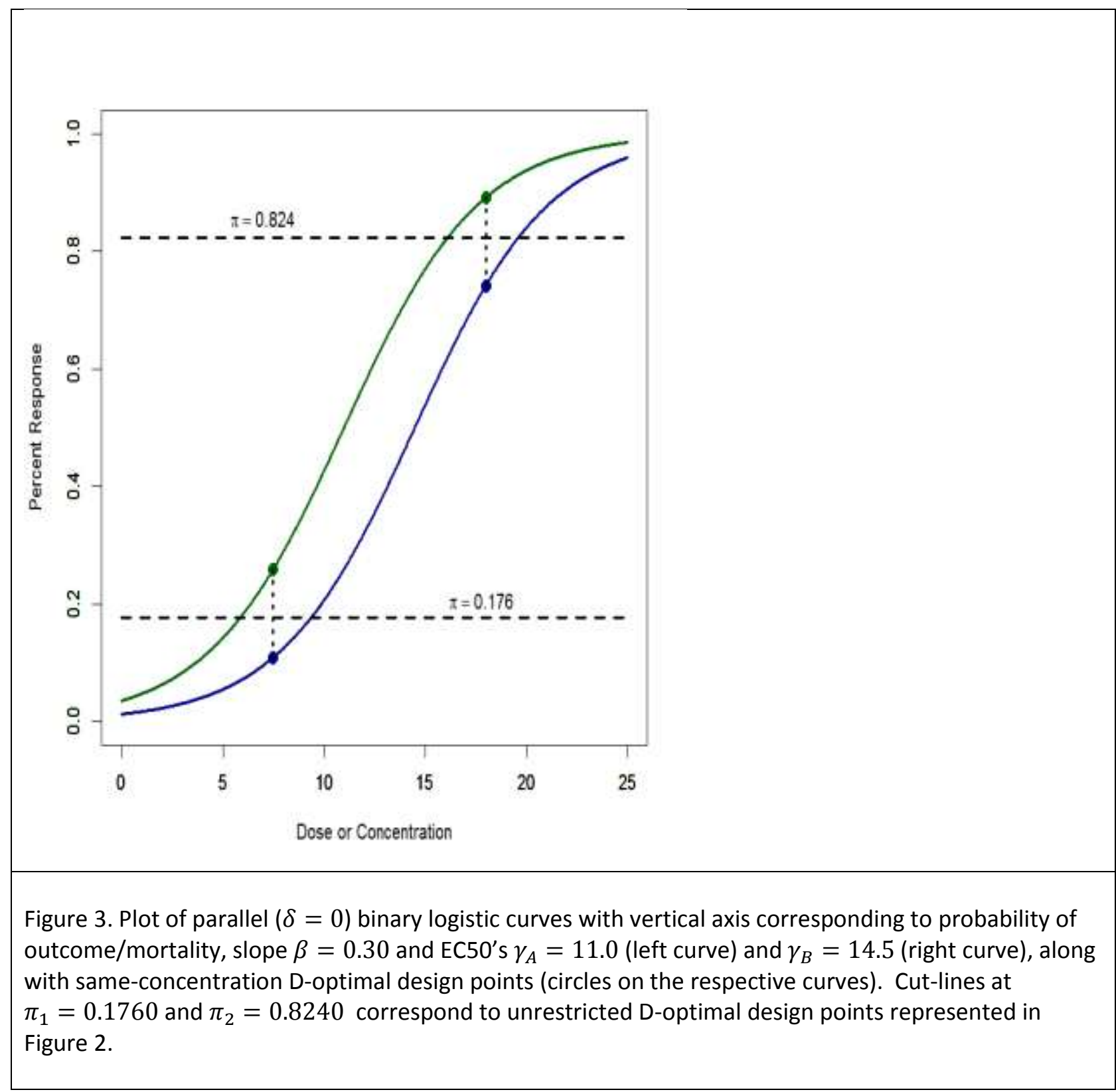

In examining the first row of Table 3 (i.e. for $\alpha=0.25$, i.e., the $D$-optimal design), note that the sameconcentration D-optimal design yields a D-efficiency (relative to the best design plotted in Figure 2) of $96.1 \%$; with a modest $3.9 \%$ information loss, this design is thus deemed to be highly efficient. Table 3 also gives analogous results for the $D_{S}(\delta)$-optimal $(\alpha=1)$ situation, where it is noted that in shifting to subset optimality, the design support points and optimal proportions again shift outward away from the EC50s. The subset efficiency of this subset design is $89.3 \%$. Our findings bear out that the patterns and observations noted here generalize to other parameter choices. 
Table 2. Same-concentration-restricted $D$ - and $D_{s}(\delta)$ designs in the parallel logistic setting: design support points and corresponding proportions, associated variance estimates for parameter values, and $D$ - and subset-efficiencies.

\begin{tabular}{|c|c|c|c|c|c|c|c|}
\hline$\alpha$ & Design & $\begin{array}{c}\text { Drug A\&B } \\
\text { support } \\
\text { points }\end{array}$ & $\begin{array}{c}\text { Drug A } \\
\text { Proportion } \\
\text { values }\end{array}$ & $\begin{array}{c}\text { Drug B } \\
\text { Proportion } \\
\text { values }\end{array}$ & $\begin{array}{c}\text { Diagonal elements of } \\
\boldsymbol{M}^{-1} \text { corresponding to } \\
\boldsymbol{\theta}^{T}=\left(\beta, \gamma_{A}, \gamma_{B}, \delta\right)\end{array}$ & $D E F F$ & $D E F F_{S}$ \\
\hline 0.25 & $D$-optimal & $\begin{array}{c}x_{1}=7.4844 \\
x_{2}=18.0156\end{array}$ & $\begin{array}{c}\pi_{1}=0.2583 \\
\pi_{2}=0.8914\end{array}$ & $\begin{array}{c}\pi_{1}=0.1086 \\
\pi_{2}=0.7417\end{array}$ & $\begin{array}{c}0.5607,154.1, \\
154.1,1.1214\end{array}$ & 0.961 & 0.731 \\
\hline \multirow{2}{*}{1.0} & $D_{S}(\delta)$ & $x_{1}=4.8600$ & $\pi_{1}=0.1368$ & $\pi_{1}=0.0526$ & $0.4587,275.6$, & 0.811 & 0.893 \\
& subset- & $x_{2}=20.6404$ & $\pi_{2}=0.9475$ & $\pi_{2}=0.8632$ & $275.6,0.9173$ & 0.963 \\
\hline
\end{tabular}

A major disadvantage of the same-concentration restriction is that the resulting near-optimal designs are observed to comprise only two support points, and thus provide no ability to check for model misspecification. As such, we now consider the following robust modification of the original unrestricted D-optimal design approach illustrated in Figure 2 and Table 1.

\subsection{Reflection Designs}

One means of obtaining robust near-optimal designs (i.e., designs with reasonably high efficiency and additional support points) in the two-logistic situation of (2) is to simply use for both drugs each of the four distinct concentrations in Figure 2 - that is, the four concentrations where - for at least one of the curves $-\pi=0.1760$ and $\pi=0.8240$. We call such designs reflection designs. For the parameter values used here, this situation is illustrated in Figure 4 . The additional reflection $\pi$ values use ex $=e^{\beta\left(\gamma_{B}-\gamma_{A}\right)}, \pi_{1}=\frac{s_{1}}{1+s_{1}}=\pi_{7}, \pi_{2}=\frac{s_{2}}{1+s_{2}}=1-\pi_{1}=\pi_{8}$ and the relations for all such parallel-curve (i.e., $\delta=0$ ) reflection design situations are as follows:

$$
\pi_{3}=\frac{e x s_{1}}{1+e x s_{1}} ; \quad \pi_{4}=\frac{e x s_{2}}{1+e x s_{2}} ; \quad \pi_{5}=\frac{s_{1}}{e x+s_{1}}=1-\pi_{4} ; \quad \pi_{6}=\frac{s_{2}}{e x+s_{2}}=1-\pi_{3}
$$

For the parameter values used here, we obtain $s_{1}=0.2137, s_{2}=4.6805$, ex $=2.858, \pi_{1}=\pi_{7}=$ $0.1760, \pi_{2}=\pi_{8}=0.8240, \pi_{3}=0.3791, \pi_{4}=0.9304, \pi_{5}=0.0696, \pi_{6}=0.6209$. 


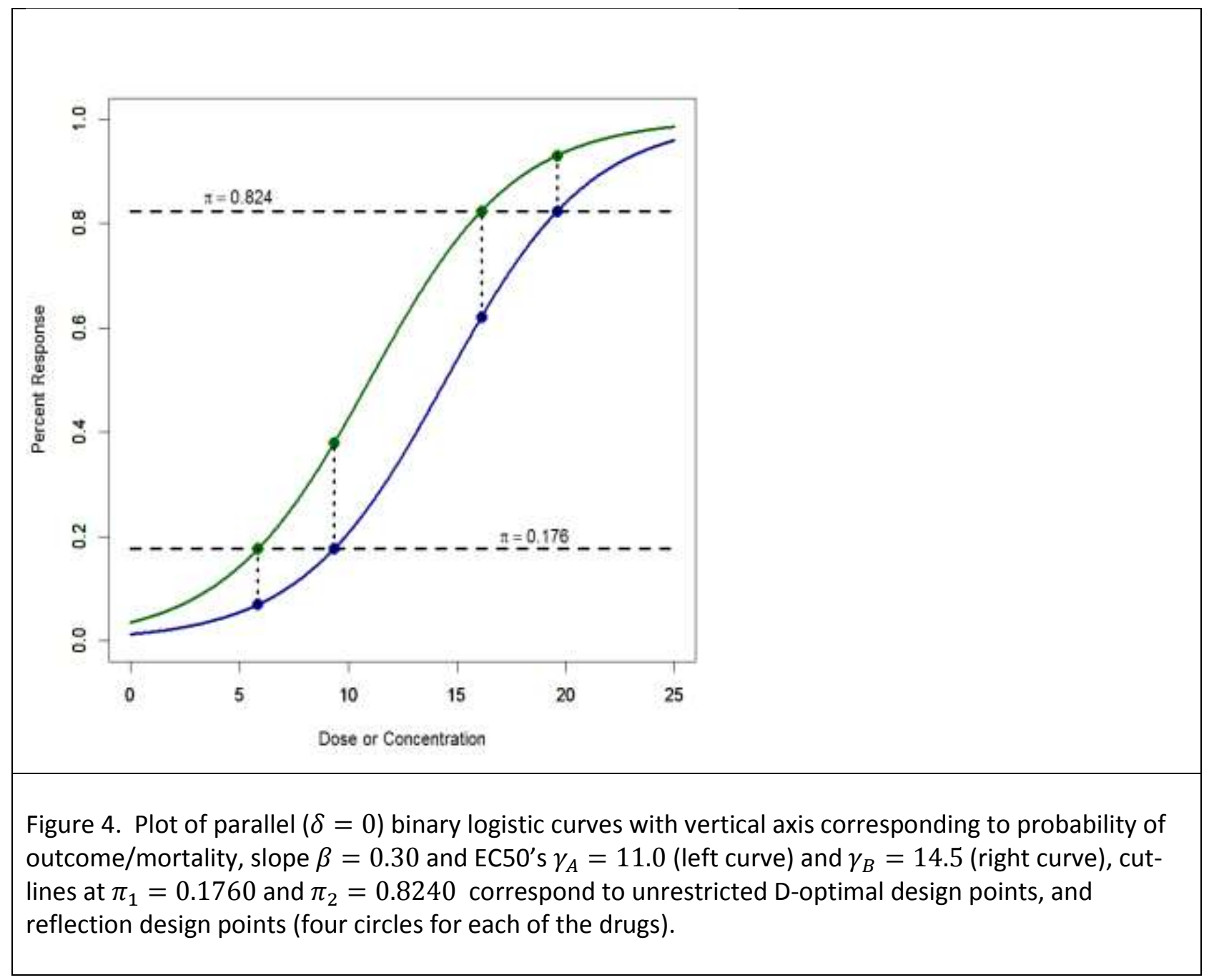

For the chosen parameter values, designs, support points, proportion values and summary statistics are given in Table 3 for reflection designs for $\alpha$ values in Equation (7) of $\alpha=0.25$ (i.e. $D$-optimality), $\alpha=0.87$ (equal-efficiency), and $\alpha=1.0$ (i.e., $D_{S}(\delta)$-optimality); note that the equal-efficiency design has been chosen so the two efficiency values, $D E F F$ and $D E F F_{S}$, are approximately equal. It is important to note that, with a $D$-efficiency of $93.2 \%$, the $D$-optimal reflection design is observed to be highly efficient - i.e., with an efficiency loss of less than $7 \%$. This design is also robust in that it provides additional support points to test for model lack-of-fit, and it is very practical to use in that scientific researchers merely need to sketch the anticipated dose-response curves for the two drugs/substances, and obtain design support points resulting from the cut lines at $\pi_{1}=0.1760$ and $\pi_{2}=0.8240$ (or thereabouts). The subset efficiency of this design is $67.1 \%$; should a researcher desire higher subset efficiency, the value of $\alpha$ could be increased to meet the researcher's objectives. For example, the equal-efficiency design ( $\alpha=0.87$ ) results in efficiencies for the full parameter vector and for the $\delta$ (difference of slopes) parameter of about $84 \%$; in this case, the proportion cut values, at $\pi_{1}=0.0951$ and $\pi_{2}=0.9049$ - or very nearly $\pi_{1}=0.10$ and $\pi_{2}=0.90$ - are also very practical to implement by researchers. As has been observed above for unconstrained and same-concentration designs, as $\alpha$ values are increased in (7) to emphasize efficient estimation of the difference-in-slopes parameter $(\delta)$, the design support points shift out (i.e., away from the EC50's). As noted in Table 3, one down-side of 
this shift is that the variability associated with these EC50 values increase here by $56 \%$ (from 151.2 to 235.7 ) for $\alpha=0.87$ and by $74 \%$ (from 151.2 to 263.8 ) for $\alpha=1.0$. We discuss implications of these results in terms of design performance, as well as overall recommendations, in Sections 5 and 6.

Table 3. Reflection $D$-optimal, equal-efficiencies, and $D_{S}(\delta)$ designs in the parallel logistic setting with parameter values given above: design support points and corresponding proportions, associated variance estimates for parameter values, and $D$ - and subset-efficiencies.

\begin{tabular}{|c|c|c|c|c|c|c|c|}
\hline$\alpha$ & Design & $\begin{array}{l}\text { Drug A\&B } \\
\text { support } \\
\text { points }\end{array}$ & $\begin{array}{l}\text { Drug A } \\
\text { Proportion } \\
\text { values } \\
\end{array}$ & $\begin{array}{l}\text { Drug B } \\
\text { Proportion } \\
\text { values } \\
\end{array}$ & $\begin{array}{c}\text { Diagonal elements of } \\
\boldsymbol{M}^{-\mathbf{1}} \text { corresponding to } \\
\boldsymbol{\theta}^{\boldsymbol{T}}=\left(\beta, \gamma_{A}, \gamma_{B}, \delta\right)\end{array}$ & $D E F F$ & $D E F F_{S}$ \\
\hline 0.25 & $D$-optimal & $\begin{array}{c}x_{1}=5.8554 \\
x_{2}=9.3554 \\
x_{3}=16.1447 \\
x_{4}=19.6447\end{array}$ & $\begin{array}{l}\pi_{1}=0.1760 \\
\pi_{2}=0.3791 \\
\pi_{3}=0.8240 \\
\pi_{4}=0.9304\end{array}$ & $\begin{array}{l}\pi_{1}=0.0696 \\
\pi_{2}=0.1760 \\
\pi_{3}=0.6209 \\
\pi_{4}=0.8240\end{array}$ & $\begin{array}{l}0.6106,151.2 \\
151.2,1.2212\end{array}$ & 0.932 & 0.671 \\
\hline 0.87 & $\begin{array}{c}\text { Equal } \\
\text { Efficiency }\end{array}$ & $\begin{array}{c}x_{1}=3.4889 \\
x_{2}=6.9888 \\
x_{3}=18.5111 \\
x_{4}=22.0111\end{array}$ & $\begin{array}{l}\pi_{1}=0.0951 \\
\pi_{2}=0.2309 \\
\pi_{3}=0.9049 \\
\pi_{4}=0.9645\end{array}$ & $\begin{array}{l}\pi_{1}=0.0355 \\
\pi_{2}=0.0951 \\
\pi_{3}=0.7691 \\
\pi_{4}=0.9049\end{array}$ & $\begin{array}{l}0.4873,235.7 \\
235.7,0.9746\end{array}$ & 0.841 & 0.841 \\
\hline 1.0 & $\begin{array}{l}D_{S}(\delta) \\
\text { subset- } \\
\text { optimal }\end{array}$ & $\begin{array}{c}x_{1}=3.0021 \\
x_{2}=6.5023 \\
x_{3}=18.9977 \\
x_{4}=22.4980\end{array}$ & $\begin{array}{l}\pi_{1}=0.0832 \\
\pi_{2}=0.2060 \\
\pi_{3}=0.9168 \\
\pi_{4}=0.9692\end{array}$ & $\begin{array}{l}\pi_{1}=0.0308 \\
\pi_{2}=0.0832 \\
\pi_{3}=0.7940 \\
\pi_{4}=0.9168\end{array}$ & $\begin{array}{l}0.4828,263.8 \\
263.8,0.9657\end{array}$ & 0.802 & 0.849 \\
\hline
\end{tabular}

\subsection{Geometric and Uniform Designs}

Noting the common-usage of geometric and uniform designs in practical settings, O'Brien (2016) and O'Brien et al. (2009) combined the D-optimality criterion with the geometric design structure of the form $x=a, a b, a b^{2} \ldots a b^{K}$ and the uniform design structure of the form $x=A, A+B, A+2 B \ldots A+$ $K B$, where $K$ is an adjustment value chosen by the researcher to provide a sufficient number of design support points. We adopt a similar approach here for the two-logistic model and, using the chosen parameter values, focus here only on the D-optimality criterion $(\alpha=0.25)$. (Nonetheless, these results generalize to other choices of $\alpha$ as well.) Results are given in Table 4 for the optimal geometric and uniform designs for $K=3$; this choice of $K$ makes these designs comparable with the 4-support-point designs given above.

Table 4. Optimal geometric and uniform $D$-optimal designs in the parallel logistic setting with parameter values given above: design support points and corresponding proportions, associated variance estimates for parameter values, and $D$ - and subset-efficiencies.

\begin{tabular}{|c|c|c|c|c|c|c|c|}
\hline$\alpha$ & \multirow{2}{*}{ Design } & $\begin{array}{c}\text { Drug A\&B } \\
\text { support } \\
\text { points }\end{array}$ & $\begin{array}{c}\text { Drug A } \\
\text { Proportion } \\
\text { values }\end{array}$ & $\begin{array}{c}\text { Drug B } \\
\text { Proportion } \\
\text { values }\end{array}$ & $\begin{array}{c}\text { Diagonal elements of } \\
\boldsymbol{M}^{-1} \text { corresponding to } \\
\boldsymbol{\theta}^{\boldsymbol{T}}=\left(\beta, \gamma_{A}, \gamma_{B}, \delta\right)\end{array}$ & $D E F F$ & $D E F F_{S}$ \\
\hline
\end{tabular}




\begin{tabular}{|c|c|c|c|c|c|c|c|}
\hline \multirow{4}{*}{0.25} & $D$-optimal & $x_{1}=6.2162$ & $\pi_{1}=0.1923$ & $\pi_{1}=0.0769$ & & & \\
& Geometric & $x_{2}=9.1428$ & $\pi_{2}=0.3642$ & $\pi_{2}=0.1670$ & $0.7640,133.1$, & 0.889 & 0.570 \\
& $a=6.216$ & $x_{3}=13.4473$ & $\pi_{3}=0.6757$ & $\pi_{3}=0.4217$ & $164.4,1.4377$ & & \\
& $b=1.471$ & $x_{4}=19.7783$ & $\pi_{4}=0.9330$ & $\pi_{4}=0.8297$ & & & \\
0.25 & D-optimal & $x_{1}=5.5755$ & $\pi_{1}=0.1642$ & $\pi_{1}=0.0643$ & & & \\
& Uniform & $x_{2}=10.3585$ & $\pi_{2}=0.4520$ & $\pi_{2}=0.2240$ & $0.6830,146.1$, & 0.902 & 0.600 \\
& $A=5.575$ & $x_{3}=15.1415$ & $\pi_{3}=0.7760$ & $\pi_{3}=0.5480$ & $146.1,1.3660$ & & \\
& $B=4.783$ & $x_{4}=19.9245$ & $\pi_{4}=0.9357$ & $\pi_{4}=0.8358$ & & & \\
\hline
\end{tabular}

As noted in Table 4, the geometric design yields slightly lower efficiencies compared with the uniform design, as well as diverse proportions (i.e., values of $\pi$ ) and unequal variance terms for the two EC50 values. The uniform design, on the other hand, results in a recognizable pattern of proportions (viz, $\pi_{1 A}+\pi_{4 B}=\pi_{2 A}+\pi_{3 B}=\pi_{3 A}+\pi_{2 B}=\pi_{4 A}+\pi_{1 B}=1$ ), and somewhat lower variance values. Both of these designs, however, perform slightly worse than the D-optimal ( $\alpha=0.25$ ) reflection design in the previous section in terms of the efficiency measures. These designs are further examined using simulations in the following section.

\section{Simulation Results}

The highlighted designs from the previous sections - and listed below for ease of comparison - were each evaluated using simulation methods with $S=5000$ simulations to assess their performance in practice and using key measures discussed below.

Table 5a. Designs examined in simulations: each design comprised $4 n$ observations for both drugs. Case I (small-to-moderate size) with $n=15$ (i.e., 60 observations for both drugs) and Case II (moderate-to-large size) with $n=30$ (i.e., 120 observations for both drugs)

\begin{tabular}{|c|l|c|l|c|}
\hline Design & \multicolumn{1}{|c|}{ Description } & Text Table & \multicolumn{1}{|c|}{ Replicates } & Support Points \\
\hline 1 & $\begin{array}{l}\text { Reflection design: } \alpha=0.87 \\
\text { (Equal Efficiency) }\end{array}$ & 3 & $\begin{array}{l}n \text { observations for each } \\
\text { drug and each support point }\end{array}$ & $\begin{array}{c}x=3.4889,6.9888, \\
18.5111,22.0111\end{array}$ \\
\hline 2 & $\begin{array}{l}\text { Reflection design: } \alpha=0.25 \\
\text { (D-optimal) }\end{array}$ & 3 & $\begin{array}{l}n \text { observations for each } \\
\text { drug and each support point }\end{array}$ & $\begin{array}{c}x=5.8554,9.3554, \\
16.1447,19.6447\end{array}$ \\
\hline 3 & $\begin{array}{l}\text { Geometric design: } \alpha=0.25 \\
\text { (D-optimal) }\end{array}$ & 4 & $\begin{array}{l}n \text { observations for each } \\
\text { drug and each support point }\end{array}$ & $\begin{array}{c}x=6.2162,9.1428, \\
13.4473,19.7783\end{array}$ \\
\hline 4 & $\begin{array}{l}\text { Uniform design: } \alpha=0.25 \\
\text { (D-optimal) }\end{array}$ & 4 & $\begin{array}{l}n \text { observations for each } \\
\text { drug and each support point }\end{array}$ & $\begin{array}{c}x=5.5755,10.3585, \\
15.1415,19.9245\end{array}$ \\
\hline 5 & $\begin{array}{l}\text { Same-concentration design: } \\
\alpha=0.25 \text { (D-optimal) }\end{array}$ & 2 & $\begin{array}{l}2 n \text { observations for each } \\
\text { drug and each support point }\end{array}$ & $x=7.4844,18.0156$ \\
\hline 6 & $\begin{array}{l}\text { Same-concentration design: } \\
\alpha=1.0 \text { (D-subset) }\end{array}$ & 2 & $\begin{array}{l}2 n \text { observations for each } \\
\text { drug and each support point }\end{array}$ & $x=4.8600,20.6404$ \\
\hline
\end{tabular}


As noted in Table 5a, our first simulation, called "Case I: 15/30", involved the small-to-moderate-sample situation of 15 replicates of each of the 4-point designs (designs 1-4) and 30 replicates of each of the 2point designs (designs 5 and 6 ) for each drug - so total sample size of 60 . This process was subsequently repeated for the moderate-to-large sample case, called "Case II: 30/60", which entailed 30 replicates of each of the 4-point designs and 60 replicates of each of the 2-point designs for each drug - i.e., total sample size of 120 . In each case, independent binary data were generated from the two-logistic situation in (2) using parameter values used above, viz, $\beta=0.30, \delta=0, \gamma_{A}=11.0, \gamma_{B}=14.5$. Simulation results for Cases I and II are given in Tables $5 b$ and $5 c$ respectively.

Table 5b. Simulations Results for Case I (15/30 study), i.e., with $n=15$ in Table $5 a$.

\begin{tabular}{|c|c|c|c|c|}
\hline Design & $\begin{array}{l}\text { Average of } \hat{\delta} \\
\text { Estimates }\end{array}$ & $\begin{array}{l}\text { Variance of } \hat{\delta} \\
\text { Estimates }\end{array}$ & $\begin{array}{l}\text { Proportion of Simulations } \\
\text { with }|\hat{\delta}|>1\end{array}$ & $\begin{array}{l}\text { Proportion of Simulations } \\
\text { with abs-value of }\left|\boldsymbol{M}^{-1}\right|>1\end{array}$ \\
\hline 1 & 0.0317 & 2.3625 & $0.0548(\approx 274 / 5000)$ & $0.0557(\approx 278 / 5000)$ \\
\hline 2 & 0.0058 & 0.0940 & $0.0014(\approx 7 / 5000)$ & $0.0014(\approx 7 / 5000)$ \\
\hline 3 & 0.0014 & 0.0251 & $0.0002(\approx 1 / 5000)$ & $0.0002(\approx 1 / 5000)$ \\
\hline 4 & -0.0013 & 0.0435 & $0.0012(\approx 6 / 5000)$ & $0.0012(\approx 6 / 5000)$ \\
\hline 5 & -0.0040 & 0.2951 & $0.0510(\approx 255 / 5000)$ & $0.0500(\approx 250 / 5000)$ \\
\hline 6 & 0.0435 & 0.8514 & $0.3325(\approx 1662 / 5000)$ & $0.3564(\approx 1782 / 5000)$ \\
\hline
\end{tabular}

The results in Table $5 b$ confirm - as do additional unreported findings - that as $\alpha$ is chosen other than the D-optimal case $(\alpha=0.25)$, estimation of the difference-of-slopes parameter $\delta$ becomes unstable; this is clearly demonstrated by Designs 1 and 6 in Table $5 b$. As noted above, in these cases, the design support points move away from the EC50 values, and thus estimation of the full parameter vector is also unstable; this is demonstrated above in large values of the generalized variance, $\left|\boldsymbol{M}^{-1}\right|$. An important empirical result observed here is that although our ultimate focus is on assessing the difference-ofslopes parameter, designs must be chosen to estimate all model parameters in order to be viable. Also, given the weak performance of the same-concentration D-optimal (two-support point) design in Design 5 , this design is also dismissed. As such, reasonable contenders include only the reflection, geometric and uniform D-optimal designs: i.e., Designs 2, 3 and 4. As samples sizes are doubled for the Case II "30/60" (i.e., Table 5c), the situation improves as expected for all designs, but clearly the most viable robust design approaches are seen to be the reflection, geometric and uniform D-optimal designs.

Table 5c. Simulations Results for Case II (30/60 study), i.e., with $n=30$ in Table 5a.

\begin{tabular}{|c|c|c|c|c|}
\hline Design & $\begin{array}{l}\text { Average of } \hat{\delta} \\
\text { Estimates }\end{array}$ & $\begin{array}{l}\text { Variance of } \hat{\delta} \\
\text { Estimates }\end{array}$ & $\begin{array}{l}\text { Proportion of Simulations } \\
\text { with }|\hat{\delta}|>1\end{array}$ & $\begin{array}{l}\text { Proportion of Simulations } \\
\text { with abs-value of }\left|M^{-1}\right|>1\end{array}$ \\
\hline 1 & -0.0033 & 0.0733 & $0.0016(\approx 8 / 5000)$ & $0.0016(\approx 8 / 5000)$ \\
\hline 2 & 0.0011 & 0.0059 & $0.0000(\approx 0 / 5000)$ & $0.0000(\approx 0 / 5000)$ \\
\hline 3 & $3.6 \mathrm{e}-06$ & 0.0069 & $0.0000(\approx 0 / 5000)$ & $0.0000(\approx 0 / 5000)$ \\
\hline 4 & -0.0014 & 0.0069 & $0.0000(\approx 0 / 5000)$ & $0.0000(\approx 0 / 5000)$ \\
\hline 5 & 0.0020 & 0.0186 & $0.0022(\approx 11 / 5000)$ & $0.0020(\approx 10 / 5000)$ \\
\hline
\end{tabular}




\section{Discussion}

The theoretical results in Section 4 as well as the empirical results in Section 5 lead us to advocate for the reflection, geometric and uniform designs introduced and illustrated in Section 4 in designing for the assessment of parallelism for the two-logistic model in (2). In terms of efficiencies, reflection designs are preferred. Furthermore, in terms of straightforward ease-of-implementation, these same reflection designs are highly favorable since as noted above the researcher merely sketches the drug/compound logistic curves and reads off design support points at the intersections with cut lines at $\pi_{1}=0.1760$ and $\pi_{2}=0.8240$. (Geometric and uniform designs generally require optimal design software.) The above simulation results confirm the favorable performance of these reflection designs even in small-tomoderate sample size situations as described above in Table 5b.. The addition of design support points when using the reflection design over the theoretical two-point D-optimal designs in Figure 2 cannot be overstressed since researchers typically wish to both efficiently estimate model parameters and check for model adequacy.

\section{References}

Abdelbasit, K.M., Plackett, R.L.: Experimental design for binary data. J. Amer. Stat. Assoc. 78, 90-98 (1983).

Atkinson, A.C.: Planning experiments to detect inadequate regression models. Biometrika 59, 275-293 (1972).

Atkinson, A.C., Donev, A. N., Tobias, R.D.: Optimum Experimental Designs, with SAS. Oxford, New York (2007)

Finney, D.J.: Probit Analysis, $3^{\text {rd }}$ Ed. Cambridge (1971)

Finney, D.J.: Statistical Method in Biological Assay, $3^{\text {rd }}$ Ed. Charles Griffin: London (1978)

Fleetwood, K., Bursa, F., Yellowlees, A.: Parallelism in practice: Approaches to parallelism in bioassays. PDA J. Pharm. Sci. Tech. 69, 248-263 (2015).

Gottschalk, P.G., Dunn, J.R.: Measuring parallelism, linearity, and relative potency in bioassay and immunoassay data. J. Biopharm. Stat. 15, 437-463, 2005.

Gupta, V., Vale, P.F.: Nonlinear disease tolerance curves reveal distinct components of host responses to viral infection. Roy. Soc. Open Sci. 4, 170342 (2017)

Jonkman, J.N., Sidak, K.: Equivalence testing for parallelism in the four-parameter logistic model. J. Biopharm. Stat. 19, 818-837 (2009).

Kalish, L.A.: Efficient design for estimation of median lethal dose and quantal dose-response curves. Biometrics 46, 737-748 (1990). 
Minkin, S.: Optimal designs for binary data. J. Amer. Stat. Assoc. 82, 1098-1103 (1987).

Novick, S.J., Yang, H., Peterson, J.J.: A Bayesian approach to parallelism testing in bioassay. Stat. Biopharm. Res. 4, 357-374 (2012).

O’Brien, T.E.: Designing for parameter subsets in Gaussian nonlinear regression models. J. Data Sci. 3, 179-197 (2005).

O'Brien, T.E.: Efficient experimental design strategies in toxicology and bioassay. Stat. Optim. Inf. Comp. 4, 99-106 (2016).

O’Brien, T.E.: Contemporary robust optimal design strategies. In Tez, M., von Rosen, D. (eds.) Trends and Perspectives in Linear Statistical Inference, pp. 165-180, Springer-Verlag: Cham, Switzerland (2018).

O'Brien, T.E., Funk, G.M.: A gentle introduction to optimal design for regression models. Amer. Stat. 57, 265-267 (2003).

O'Brien, T.E. and Lim, C.: New challenges and strategies in robust optimal design for multicategory logit modelling. In Chen, D., Jin, Z., Li, G., Li, Y., Liu, A. and Zhao, Y. (eds.), New Advances in Statistics and Data Science, pp.61-74, Springer-Verlag: Cham, Switzerland (2018).

O'Brien, T.E., Rawlings, J.O.: A non-sequential design procedure for parameter estimation and model discrimination in nonlinear regression models. J. Stat. Plan. Inf. 55, 77-93 (1996).

O'Brien, T.E., Chooprateep, S., Homkham, N.: Efficient geometric and uniform design strategies for sigmoidal regression models. S. Afric. Stat. J. 43, 49-83 (2009).

O’Brien, T.E., Jamroenpinyo, S., Bumrungsup, C.: Curvature measures for nonlinear regression models using continuous designs with applications to optimal design. Involve J. Math. 3, 317-332 (2010).

Rich, I.N.: Potency, proliferation and engraftment potential of stem cell therapeutics: the relationship between potency and clinical outcome for hematopoietic stem cell products. J. Cell Sci. Ther. S13:001, doi: 10.4172/2157-7013.S13-001

Sidak, K., Jonkman, J.N.: Testing for parallelism in the heteroskedastic four-parameter logistic model. J. Biopharm. Stat. 26, 250-268 (2016).

Wheeler, M.W., Park, R.M., Bailer, A.J.: Comparing median lethal concentration values using confidence interval overlap or ratio tests. Envir. Tox. Chem. 25, 1441-1444 (2006).

Yang, H., Zhang, L.: Evaluations of parallelism testing methods using ROC analysis. Stat. Biopharm. Res. 4, 162-173 (2012).

Yang, H., Kim, H.J., Zhang, L., Strouse, R.J., Schenerman, M., Jiang, X-R.: Implementation of parallelism testing for four-parameter logistic model in bioassays. PDA J. Pharm. Sci. Tech. 66, 262-269 
(2012). 